\title{
A New Nonlinear Active Disturbance Rejection Control for the Cable Car System to Restrain the Vibration
}

\author{
Xinyan Hu $\mathbb{D}$ and Lina Li $\mathbb{D}$ \\ College of Intelligent Equipment, Shandong University of Science and Technology, Tai'an, Shandong 271019, China \\ Correspondence should be addressed to Xinyan Hu; huxinyan@sdust.edu.cn
}

Received 29 July 2020; Revised 24 August 2020; Accepted 1 September 2020; Published 16 September 2020

Academic Editor: Shubo Wang

Copyright ( $) 2020$ Xinyan Hu and Lina Li. This is an open access article distributed under the Creative Commons Attribution License, which permits unrestricted use, distribution, and reproduction in any medium, provided the original work is properly cited.

\begin{abstract}
The safety of the cable car system is very important for the lives of the people. But, it is easily affected by the environment such as the wind which causes the cable car system to have strong vibration disturbance, thus degrading the safety of the cable car system. In this paper, a new nonlinear active disturbance rejection control (ADRC) is proposed to restrain the vibration of the cable car. First, a new two-mass-spring system model is utilized to establish the cable car system model. The new translation vibration nonlinear model is derived by a linear-invariant two-mass-spring system. Then, a special nonlinear fal $(\bullet)$ is designed to restrain the vibration, and a new high-order nonlinear ADRC is presented for the cable car system. Finally, simulation results verify the feasibility and accuracy of the proposed model.
\end{abstract}

\section{Introduction}

Cable car is a means of transportation, which is erected on the slopes of the scenic area to transport tourists. There are many types of cable cars. This paper mainly studies the ropeway cable car. It is susceptible to strong interference, such as changes in the number of passengers, airflow, and changes in the characteristics of the cable car installation system, all of which affect the safe operation of the ropeway cable car. The ropeway cable car system is mainly composed of the driving device, load-bearing rope, traction rope, carriage, walking trolley, and anchoring drum. It is a typical complex electromechanical system with the characteristics of time-varying parameters, strong coupling, and nonlinearity. The driving device and the walking trolley are the core control components of the cable car control system, as well as the main factors affecting the cable car vibration. During normal operation, this part of the control system is a flexible connection, variable mass, variable speed, and variable stiffness motion control system.

As far as the control mechanism is concerned, the vibration problem belongs to the category of motion control and is caused by the resonance mode of the system; therefore, the two have great similarities. Thus, the vibration problem and the resonance mode can be attributed to the same type of problem. The resonance mode is the inherent internal dynamic characteristics of the system. The resonance mode changes with the change of the system parameters. When the natural frequency of the resonance is close to or overlaps the system bandwidth, it may cause mechanical resonance, causing oscillation and instability of the system. It makes the personal safety of cable car tourists greatly threatened. Based on this, how to suppress mechanical resonance problem in the ropeway cable car system has become the research direction of more and more experts and scholars. To deal with the resonance problem, on the one hand, the mechanical structure of the ropeway cable car system can be improved and on the other hand, the control compensation of the control system can also be started. Therefore, studying the control methods and controller design of complex control systems with time-varying parameters, strong coupling, multivariate factors, and other nonlinear factors can effectively improve the control effect of the ropeway cable car system, which is of great practical significance.

At present, the control method used in the ropeway cable car system is still based on traditional PID and improved 
PID. However, the control effect of this traditional single control method is increasingly unable to meet the actual control requirements of the complex control system, and it is difficult to solve the problems of system oscillation in the cable car system. Siemens, Yaskawa, and Panasonic have all adopted notch filters for simple compensation or correction of the servo system. This passive suppression method can effectively suppress mechanical resonance problems in the system, but it is necessary to know the resonant frequency of the ropeway cable car system when using the notch filter to compensate the system, which makes it more complicated to suppress the mechanical resonance of the system. Active disturbance rejection control treats all uncertain items and disturbance items of the system as total disturbances. Regardless of the resonant frequency of the cable car system, they can be treated as total disturbances, which have certain advantages.

In order to solve the control compensation of the vibration problem in the electromechanical system, many domestic and foreign scholars have carried out a lot of research work. Wie and Bernstein $[1,2]$ refined the vibration and disturbance control problems of translational motion control and summarized them as benchmark problems expressed by the two-mass-spring system. In four types of design problems, they pointed out the performance requirements that the controller design should meet. For example, "for a unit impulse disturbance exerted on body 1 and/or body 2 , the controlled output $\left(z=x_{2}\right)$ has a settling time of about $15 \mathrm{~s}$ for the nominal system with $m_{1}=m_{2}=k=1$." Many scholars proposed many solutions based on different control technologies after the benchmark problem was proposed, such as fuzzy control, robust optimal control, sliding mode control, adaptive control, $H-\infty$ optimal control, and other advanced algorithms and achieved the expected results [3-22]. The active disturbance rejection control (ADRC) has attracted more and more attention because it does not rely on the precise mathematical model of the controlled object, and its algorithms are simple and easy to implement in engineering [23]. Han Zhang designed a fifth-order LADRC controller aiming at the fourth-order object model of the two-mass-spring system and achieved good control results [24].

This paper analyzes the vibration model aiming at the vibration problem of the ropeway cable car system and designs a new nonlinear ADRC to suppress various uncertain nonlinear factors in the system [25-36]. Not only can the dynamic characteristics of the control system be improved, but also the mechanical resonance problem in the ropeway cable car system can be suppressed, and good results have been achieved.

The other organization content of this paper is arranged as follows: the second part completes the analysis of the cable car vibration model and derives the general expression of the cable car vibration model; the third part analyzes and designs the algorithm of nonlinear active disturbance rejection controller (NLADRC) aiming at the control goal of the cable car vibration; the fourth part applies a certain disturbance according to the cable car model which has been established and the control algorithm which has been designed and completes the verification through simulation technology; the fifth part gives the research conclusions of this paper and follow-up research objectives.

\section{Problem Formulations}

The cable car driving device pulls the walking trolley on the load-bearing rope through the traction cable so that the carriage reciprocates around the anchor drum to transport tourists. According to the motion form of the carriage carrier, it can be regarded as a translational motion control. The common vibration and disturbance suppression problems in the translational motion control can be reduced to a benchmark problem expressed by a two-mass-spring system. Assuming that the mass of the traction cable and its accessories is $M_{1}$ and the mass of the walking trolley and the carriage is $M_{2}$, considering the friction and the flexible connection device of the traction cable, it can be equivalent to a spring with a coefficient of $K$ and a translational damper with a coefficient of $C$ in parallel. Consider that, under ideal conditions first, $M, K$, and $C$ are constants and then analyze the model. The schematic diagram is shown in Figure 1.

Among them, the mass of the traction device is $M_{1}$ and its displacement is $q(t)$ under the action of the control force $u(t)$ and the mass of the weight thing is $M_{2}$ and its displacement is $c(t)$ under the action of the equivalent spring and translation damper.

According to Newton's second law and Hooke's law, ignoring friction, the force condition on the mass block $M_{1}$ is shown as follows:

$$
M_{1} \ddot{q}=-K(q-c)-C(\dot{q}-\dot{c})+u(t)
$$

The force condition of the mass block $M_{2}$ is shown as follows:

$$
M_{2} \ddot{q}=-K(c-q)-C(\dot{c}-\dot{q}) .
$$

Ignoring noise and interference, we set $x_{1}=q, x_{2}=c$, $x_{3}=\dot{q}, x_{4}=\dot{c}$, and $y=x_{2}$. The state space representation of the system can be obtained as follows:

$$
\left\{\begin{array}{l}
\dot{x}_{1}=x_{3}, \\
\dot{x}_{2}=x_{4}, \\
\dot{x}_{3}=-\frac{K}{M_{1}} x_{1}+\frac{K}{M_{1}} x_{2}-\frac{C}{M_{1}} x_{3}+\frac{C}{M_{1}} x_{4}+\frac{1}{M_{1}} u \\
\dot{x}_{4}=\frac{K}{M_{2}} x_{1}-\frac{K}{M_{2}} x_{2}+\frac{C}{M_{2}} x_{3}-\frac{C}{M_{2}} x_{4}, \\
y=x_{2} .
\end{array}\right.
$$

The state space representation represented by the vector matrix is shown as follows:

$$
\left\{\begin{array}{l}
\dot{x}(t)=A x(t)+B u(t) \\
y(t)=C x(t)+D u(t)
\end{array}\right.
$$




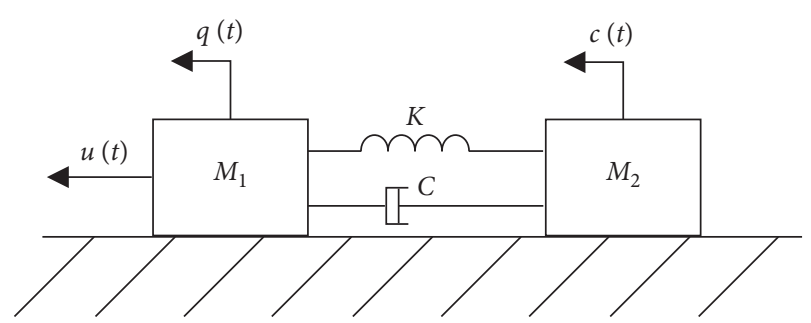

FIGURE 1: The schematic diagram of the two-mass-spring system.

Among them,

$$
\begin{aligned}
& \mathbf{A}=\left[\begin{array}{cccc}
0 & 0 & 1 & 0 \\
0 & 0 & 0 & 1 \\
-\frac{K}{M_{1}} & \frac{K}{M_{1}} & -\frac{C}{M_{1}} & \frac{C}{M_{1}} \\
\frac{K}{M_{2}} & -\frac{K}{M_{2}} & \frac{C}{M_{2}} & -\frac{C}{M_{2}}
\end{array}\right], \\
& \mathbf{B}=\left[\begin{array}{c}
0 \\
0 \\
\frac{1}{M_{1}} \\
0
\end{array}\right], \\
& \mathbf{C}=\left[\begin{array}{lll}
0 & 1 & 0
\end{array}\right], \\
& \mathbf{D}=0 .
\end{aligned}
$$

The above-deduced formula is the state space model of a linear system under ideal conditions, which is similar to a fourth-order linear time-invariant system. Referring to equation (3), ignoring noise and disturbance, and considering the nonlinear model of the system in a more general sense, the following equation can be derived:

$$
\left\{\begin{array}{l}
\dot{x}_{1}=x_{3}, \\
\dot{x}_{2}=x_{4}, \\
\dot{x}_{3}=f_{1}\left(x_{1}, x_{2}, x_{3}, x_{4}\right)+b u, \\
\dot{x}_{4}=f_{2}\left(x_{1}, x_{2}, x_{3}, x_{4}\right), \\
y=x_{2} .
\end{array}\right.
$$

Note that among them, $\dot{x}_{3}=f_{1}\left(x_{1} x_{2} x_{3} x_{4}\right)+b u$ can be written in a more general expression as $\dot{x}_{3}=f_{1}\left(x_{1} x_{2} x_{3} x_{4}, u\right)$. For the convenience of analysis, this equation is written as equation (6)in this paper.

Through the analysis of the two-mass-spring system, a system expression such as equation (6) is established, which is used to analyze and design the system controller later.

\section{Controller Design}

3.1. Relative Order of the System. According to the control system shown in equation (6), the force acting on the mass block $M_{1}$ is the control input $u$ of the open-loop system and the displacement of the mass block $M_{2}$ is the controlled output $y$. The purpose of control is to eliminate the oscillation as soon as possible by adjusting the control input $u$ and the controlled output $y$ to track the system setting value $v$ as soon as possible, thereby suppressing vibration.

There are many "integrator" paths from the control input $u$ to the controlled output $y$, and the path with the least number of integrators is the relative order of the system. The relative order of the system determines the order of the active disturbance rejection controller. The path structure diagram of the system integrator is shown in Figure 2.

Here, there are ten integrator paths from the control input $u$ to the controlled output $y$, such as the following:

The first path is $u \longrightarrow \int_{3} \longrightarrow \int_{2} \longrightarrow \int_{4} \longrightarrow \int_{2} \longrightarrow$ $y$. There are three integrators.

The second path is $u \longrightarrow \int_{3} \longrightarrow \int_{1} \longrightarrow f_{2} \longrightarrow$ $\int_{4} \longrightarrow \int_{2} \longrightarrow y$. There are four integrators.

The third path is $u \longrightarrow \int_{3} \longrightarrow f_{1} \longrightarrow \int_{3} \longrightarrow$ $f_{2} \longrightarrow \int_{4} \longrightarrow \int_{2} \longrightarrow y$. There are five integrators. The fourth path is $u \longrightarrow \int_{3} \longrightarrow \int_{1} \longrightarrow f_{1} \longrightarrow$ $\int_{3} \longrightarrow f_{2} \longrightarrow \int_{4} \longrightarrow \int_{2} \longrightarrow y$. There are five integrators.

After analyzing the integrator path, the least number of integrators in the path is 3 , namely, the first path, and the relative order of the system is determined to be 3 .

3.2. Analysis of Algorithms. According to the open-loop system shown in equation (6), set

$$
\left\{\begin{array}{l}
z_{1}=x_{2}, \\
z_{2}=x_{4}, \\
z_{3}=f_{2} .
\end{array}\right.
$$

Then,

$$
\left\{\begin{aligned}
\dot{z}_{1} & =\dot{x}_{2}=z_{2}, \\
\dot{z}_{2} & =\dot{x}_{4}=z_{3}, \\
\dot{z}_{3} & =\dot{f}_{2} \\
& =\frac{\partial f_{2}}{\partial x_{1}} \dot{x}_{1}+\frac{\partial f_{2}}{\partial x_{2}} \dot{x}_{2}+\frac{\partial f_{2}}{\partial x_{3}} \dot{x}_{3}+\frac{\partial f_{2}}{\partial x_{4}} \dot{x}_{4} \\
& =w+b_{0} u .
\end{aligned}\right.
$$

Among them, 


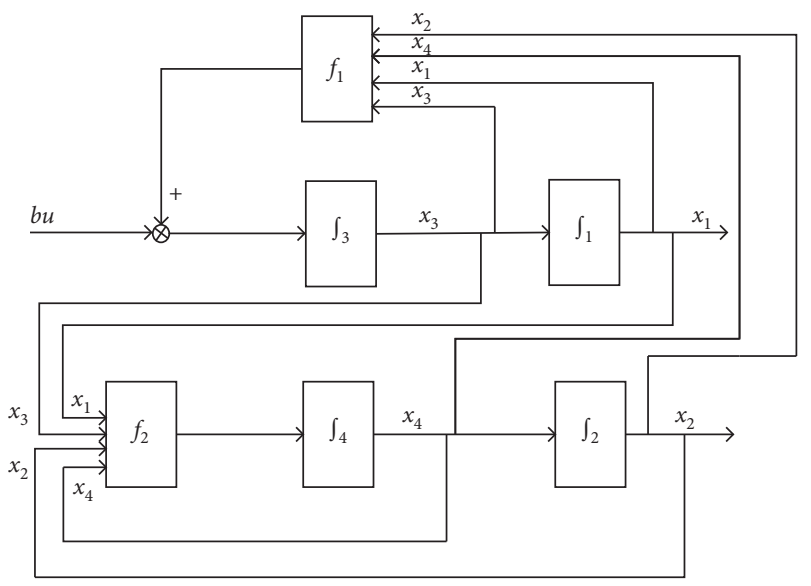

Figure 2: The schematic diagram of the integrator path.

$$
\begin{aligned}
w & =\frac{\partial f_{2}}{\partial x_{1}} \dot{x}_{1}+\frac{\partial f_{2}}{\partial x_{2}} \dot{x}_{2}+\frac{\partial f_{2}}{\partial x_{3}} f_{1}+\frac{\partial f_{2}}{\partial x_{4}} \dot{x}_{4} \\
b_{0} & =\frac{\partial f_{2}}{\partial x_{3}} b .
\end{aligned}
$$

In equation (8), $w$ is the unknown part of the system, which covers the uncertain items in the system. For example, if disturbances $w_{1}$ or $w_{2}$ are applied to two mass blocks, respectively, this part of the uncertain items can also be included in $w . w$ is the total disturbance of the system, which can be estimated in real time through ESO. $b_{0}$ is the compensation factor that determines the strength of the compensation, which can be used to adjust parameters during simulation.

According to the previous analysis and combined with the characteristics of the system, the ADRC controller structure is designed as shown in Figure 3. ADRC controller mechanism: ESO estimates the system state estimation value and the system total disturbance estimation values $z_{1} \sim z_{4}$ in real time according to the output information $y$ and input control $u$; tracking differentiator (TD) can arrange the transition process of the setting value $v$, and the differential signals of each order $v_{1} \sim v_{3}$ of $v$ are obtained at the same time; if the system error is defined as $e_{i}=v_{i}-z_{i}(i=1,2,3)$, using the system error, LSEF calculates the control law $u_{0}$ to satisfy the control objective requirements.

3.2.1. Tracking Differentiator (TD). Tracking differentiator (TD) can arrange the transition process for the input signal $v$ and calculate the approximate differential signals of each order of the input signal at the same time. In the two-massspring system, the zero-order, first-order, second-order, and third-order differential signals of the input signal $v$ are required, and the input signal $v$ is sent to the tracking differentiator to obtain the differential signals of each order of the input signal $v$.

The differential signal of each order of the input signal $v$ can be obtained by using the following state space representation:

$$
\left\{\begin{array}{l}
\dot{v}_{1}=v_{2}, \\
\dot{v}_{2}=v_{3}, \\
\dot{v}_{3}=v_{4}, \\
\dot{v}_{4}=f(v, \dot{v}, \ddot{v}, \dddot{v}) .
\end{array}\right.
$$

Using the nonlinear state observer, if the state of $v_{i}$ is observed, the differential signals of each order of the input signal $v$ can be obtained. For this reason, a high-order tracking differentiator is designed using the fal $(\bullet)$ function, and its algorithm is as follows:

$$
\left\{\begin{array}{l}
\varepsilon=v_{1}-v, \\
f \varepsilon=\mathrm{fal}\left(\varepsilon, \frac{1}{2}, \delta\right), \\
f \varepsilon_{1}=\mathrm{fal}\left(\varepsilon, \frac{1}{4}, \delta\right), \\
f \varepsilon_{2}=\mathrm{fal}\left(\varepsilon, \frac{1}{8}, \delta\right), \\
\dot{v}_{1}=v_{2}-\ell_{01} \varepsilon, \\
\dot{v}_{2}=v_{3}-\ell_{02} f \varepsilon, \\
\dot{v}_{3}=v_{4}-\ell_{03} f \varepsilon_{1}, \\
\dot{v}_{4}=-\ell_{04} f \varepsilon_{2} .
\end{array}\right.
$$

After equation (11) is discretized, the algorithm is obtained as follows:

$$
\left\{\begin{array}{l}
\varepsilon=v_{1}-v \\
f \varepsilon=\operatorname{fal}\left(\varepsilon, \frac{1}{2}, \delta\right) \\
f \varepsilon_{1}=\operatorname{fal}\left(\varepsilon, \frac{1}{4}, \delta\right) \\
f \varepsilon_{2}=\operatorname{fal}\left(\varepsilon, \frac{1}{8}, \delta\right) \\
v_{1}=v_{1}+h\left(v_{2}-\ell_{01} \varepsilon\right) \\
v_{2}=v_{2}+h\left(v_{3}-\ell_{02} f \varepsilon\right) \\
v_{3}=v_{3}+h\left(v_{4}-\ell_{03} f \varepsilon_{1}\right) \\
v_{4}=v_{4}+h\left(-\ell_{04} f \varepsilon_{2}\right)
\end{array}\right.
$$




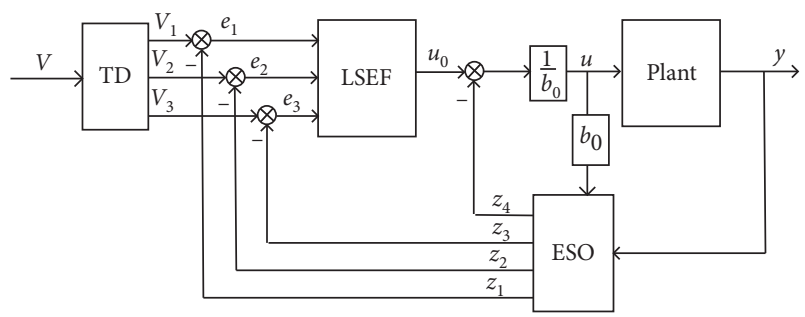

FIgUre 3: The schematic diagram of the NLADRC.

Among them, $h$ is the sampling step size; $\delta$ is the parameter that determines the speed of tracking, which can be taken as a multiple of $h ; \varepsilon$ is the tracking error; $\ell_{i}$ is the parameter of observer; the zero-order, first-order, secondorder, and third-order differential signals of the input signal $v$ are $v_{1} \longrightarrow v, v_{2} \longrightarrow \dot{v}, v_{3} \longrightarrow \ddot{v}$, and $v_{4} \longrightarrow \dddot{v}$; and the $\mathrm{fal}(\bullet)$ function is a special nonlinear structure function, which has the characteristics of fast convergence and filtering. Using the symbolic function, its expression is as follows:

$$
\left\{\begin{array}{l}
s=\left(\frac{\operatorname{sign}(\varepsilon+\delta)-\operatorname{sign}(\varepsilon-\delta)}{2}\right) \\
\operatorname{fal}(\varepsilon, \alpha, \delta)=\frac{\varepsilon}{\delta^{1-\alpha}} s+|\varepsilon|^{\alpha} \operatorname{sign}(\varepsilon)(1-s) .
\end{array}\right.
$$

Here, $\alpha \in[0,1], \delta>0$.

In equation (13), $s$ is the interval function of the tracking error $\varepsilon$, and the interval is $\varepsilon \in[-\delta, \delta]$, and $1-s$ is the function with the interval of $\varepsilon \notin[-\delta, \delta]$; when the tracking error $\varepsilon$ is small, the gain of the fal $(\bullet)$ function is $1 /\left(\delta^{1-\alpha}\right)$; when the tracking error $\varepsilon$ is large, it converges according to the power function to realize the nonlinear automatic adjustment of "small error with large gain, large error with small gain," thereby reducing oscillation and rapid convergence.

3.3. Extended State Observer (ESO). According to equation (8), the real-time action of $w$ acting on the open-loop system is expanded into a new state $z_{4}, z_{4}=w$, namely,

$$
\dot{z}_{4}=\dot{w} .
$$

Then, the system shown in equation (8) can be expanded into a new control system form as follows:

$$
\left\{\begin{array}{l}
\dot{z}_{1}=z_{2}, \\
\dot{z}_{2}=z_{3}, \\
\dot{z}_{3}=z_{4}+b_{0} u, \\
\dot{z}_{4}=\dot{w}=w_{0}, \\
y=z_{1} .
\end{array}\right.
$$

For system (15), a nonlinear state observer as shown in equation (16) can be established:

$$
\left\{\begin{array}{l}
\epsilon=z_{1}-y, \\
f \epsilon=\mathrm{fal}\left(\epsilon, \frac{1}{2}, \delta\right), \\
f \epsilon_{1}=\mathrm{fal}\left(\varepsilon, \frac{1}{4}, \delta\right), \\
f \epsilon_{2}=\mathrm{fal}\left(\varepsilon, \frac{1}{8}, \delta\right), \\
\dot{z}_{1}=z_{2}-\beta_{01} \epsilon, \\
\dot{z}_{2}=z_{3}-\beta_{02} f \epsilon, \\
\dot{z}_{3}=z_{4}-\beta_{03} f \epsilon_{1}+b_{0} u, \\
\dot{z}_{4}=-\beta_{04} f \epsilon_{2} .
\end{array}\right.
$$

After equation (16) is discretized, the following expression can be obtained:

$$
\left\{\begin{array}{l}
\epsilon=z_{1}-y, \\
f \epsilon=\mathrm{fal}\left(\epsilon, \frac{1}{2}, \delta\right), \\
f \epsilon_{1}=\mathrm{fal}\left(\epsilon, \frac{1}{4}, \delta\right), \\
f \epsilon_{2}=\mathrm{fal}\left(\epsilon, \frac{1}{8}, \delta\right) \\
z_{1}=z_{1}+h\left(z_{2}-\beta_{01} \epsilon\right), \\
z_{2}=z_{2}+h\left(z_{3}-\beta_{02} f \epsilon\right), \\
z_{3}=z_{3}+h\left(z_{4}-\beta_{03} f \epsilon_{1}+b_{0} u\right), \\
z_{4}=z_{4}-h\left(\beta_{04} f \epsilon_{2}\right) .
\end{array}\right.
$$

Among them, $h$ is the sampling step size and $\beta_{01}, \beta_{02}, \beta_{03}$, and $\beta_{04}$ are the parameters of the extended state observer, and the parameters of the extended state observer can adopt the parameter sequence closely related to Fibonacci sequences, which is inherited. The parameter sequences of the extended state observer are shown in Table 1:

According to equations (15) and (16), the ESO observation error equation can be obtained as follows:

$$
\left\{\begin{array}{l}
\dot{\epsilon}_{1}=\epsilon_{2}-\beta_{01} \epsilon, \\
\dot{\epsilon}_{2}=\epsilon_{3}-\beta_{02} f \epsilon, \\
\dot{\epsilon}_{3}=\epsilon_{4}-\beta_{03} f \epsilon_{1}, \\
\dot{\epsilon}_{4}=-\beta_{04} f \epsilon_{2}-w_{0},
\end{array}\right.
$$

where $f \varepsilon_{i}$ is a function of $f a l(\bullet)$, which is a function of $\varepsilon$. When the system is stable, all $\varepsilon_{i}=0$. According to equation (13), as long as $\beta_{04}$ is sufficiently larger than $w_{0}$, all errors will be small enough. ESO parameters are independent of system 
TABle 1: Parameter sequences.

\begin{tabular}{lc}
\hline Item & \\
\hline$\beta_{01}$ & $1 / 1^{0} h$ \\
$\beta_{02}$ & $1 / 3 h^{1} h$ \\
$\beta_{03}$ & $2 / 8 h^{2} h$ \\
$\beta_{04}$ & $5 / 13 h^{3} h$ \\
\hline
\end{tabular}

parameters. As long as the $\beta_{i}$ parameters are selected appropriately, the real-time state of the object can be well estimated.

3.4. Linear State Error Feedback (LSEF). From the system shown in equation (15), $\dddot{z}=w+b_{0} u$, set $w=z_{4}$. According to the input and output signals $u$ and $y$ of the system, the special nonlinear function fal $(\bullet)$ can be used to effectively estimate the total disturbance $w$ of the system. Take $u=$ $\left(u_{0}-w\right) / b_{0} \quad$ so that the system becomes $\dddot{z}=w+b_{0}\left(\left(u_{0}-w\right) / b_{0}\right)=u_{0}$; that is, the system becomes a linear integrator series system:

$$
\dddot{z}=u_{0} \text {. }
$$

Comparing with the original system, it is relatively easy to deal with the linear integrator series system. It can adopt the linear combination method or nonlinear combination method. During the operation of the system, it is sufficient to appropriately apply control forces to make $e_{1}, e_{2}$, and $e_{3}$ attenuate to zero:

$$
\begin{aligned}
& \because \quad e=v-z \Longrightarrow z=v-e, \\
& \therefore \quad \dddot{z}=u_{0}=\dddot{v}-\dddot{e} .
\end{aligned}
$$

The control quantity $u_{0}$ is taken as the state feedback of the error $e$, and its expression is as follows:

$$
u_{0}=k_{1} e_{1}+k_{2} e_{2}+k_{3} e_{3}+\dddot{v} .
$$
obtain

Substituting equation (21) into equation (20), we can

$$
\dddot{e}=-k_{1} e_{1}-k_{2} e_{2}-k_{3} e_{3}=-k_{1} e-k_{2} \dot{e}-k_{3} \ddot{e} .
$$

Equation (22) represents the differential equation form of the closed-loop system. If the conditions $k_{1}>0, k_{2}>0, k_{3}>0$, and $k_{2} k_{3}>k_{1}$ are satisfied, then the system is stable, so that $e \Rightarrow 0$, and the control purpose is achieved.

According to the above analysis, using the system errors $e_{1}, e_{2}$, and $e_{3}$ and the linear state error feedback control law, the control algorithm is designed as follows:

$$
\left\{\begin{array}{l}
e_{1}=v_{1}-z_{1}, \\
e_{2}=v_{2}-z_{2}, \\
e_{3}=v_{3}-z_{3}, \\
u_{0}=\beta_{1} e_{1}+\beta_{2} e_{2}+\beta_{3} e_{3}+\dddot{v} .
\end{array}\right.
$$

If the input signal $v$ is a constant or an indeterminate value (such as follow-up adjustment), then we can set $\dddot{v}=0$ and generally set $\dddot{v}=0$. In equation (23), according to the actual project, only the $\beta_{i}$ parameter combination needs to be adjusted.

3.5. Design of Algorithms. Through the previous analysis, the NLADRC algorithm in this paper mainly includes three parts: TD, LSEF, and ESO. The complete algorithm is as follows:

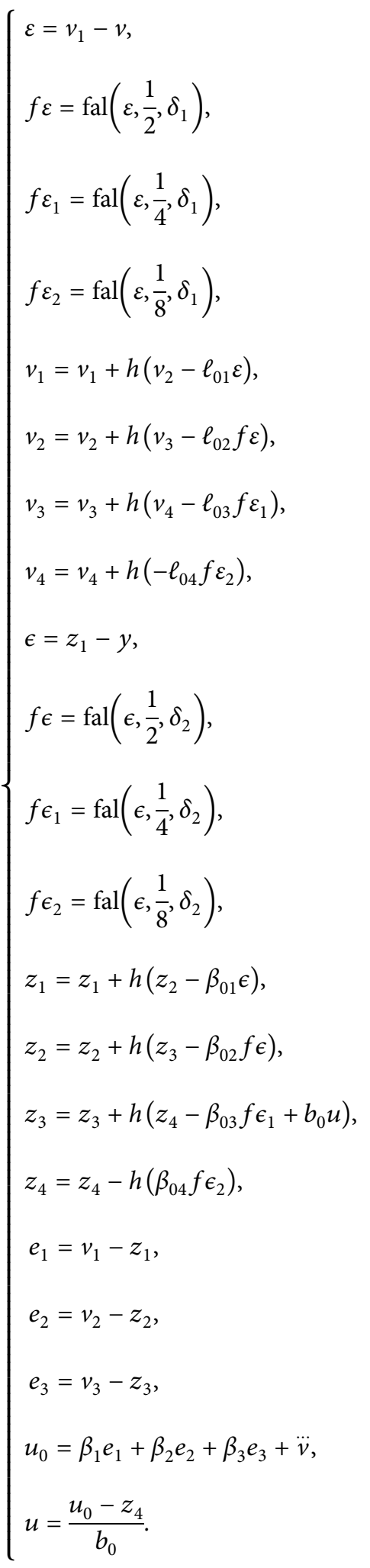




\section{Simulation}

In this paper, for the two-mass-spring system represented using equation (3), take $K=C=M_{1}=M_{2}=1$ to complete the simulation. In order to verify the influence of the disturbance on the system, the external disturbances $d_{1}$ and $d_{2}$ are applied to the two mass blocks $M_{1}$ and $M_{2}$, respectively. In order to meet the needs of simulation, the state space representation is established as follows:

$$
\left\{\begin{array}{l}
\dot{x}_{1}=x_{3}, \\
\dot{x}_{2}=x_{4} \\
\dot{x}_{3}=-x_{1}+x_{2}-x_{3}+x_{4}+d_{1}+b u \\
\dot{x}_{4}=x_{1}-x_{2}+x_{3}-x_{4}+d_{2}, \\
y=x_{2} .
\end{array}\right.
$$

4.1. TD Simulation. This paper uses the discretization form of equation (12)'s TD algorithm to analyze the zero-order, first-order, second-order, and third-order differential signals of the input signal $v$ when the input signal $v$ is a periodic square wave signal and a sine signal and verify the correctness and accuracy of the algorithm. The values of parameters in the algorithm are shown in Table 2.

When the input signal $v$ is a periodic square wave signal, see Figure 4. In Figure 4, the input signal $v$, the zero-order differential signal $v^{(0)}$, and the first-order differential signal $v^{(1)}$ are displayed.

When the input signal $v$ is a sine signal $\sin (0.1 t)$, see Figure 5. In Figure 5, the input signal $v$, the zero-order differential signal $v^{(0)}$, and the first-order differential signal $v^{(1)}$ are displayed.

When the input signal changes to $\sin (t)$ and $\sin (10 t)$, respectively, the TD output waveforms are shown in Figures 6 and 7, respectively. Through the waveforms observation of the input signal $v$ and the zero-order differential signal $v^{(0)}$, we can find that even if the frequency of the input signal changes greatly, the TD output signal can quickly follow the change and the robustness is strong.

The simulation results show that the designed TD tracking algorithm can quickly track the zero-order, firstorder, second-order, and third-order differential signals of the input signal, with good accuracy and robustness.

4.2. ESO Simulation. This paper uses the discretization form of the ESO algorithm in equation (17) to analyze and compare the output state signal of the observer with the state signal to be observed and verify the correctness and accuracy of the algorithm. The values of parameters in the algorithm are shown in Table 3.

In Figure 8, the observer output state signal $z_{1}$ and the state signal to be observed $x_{2}$ are displayed.
TABLE 2: TD parameter table.

\begin{tabular}{lc}
\hline Item & \\
\hline$h$ & 0.01 \\
$\delta_{1}$ & $h$ \\
$\ell_{01}$ & $1 / 1^{0} h$ \\
$\ell_{02}$ & $1 / 3 h^{1} h$ \\
$\ell_{03}$ & $2 / 8 h^{2} h$ \\
$\ell_{04}$ & $5 / 13 h^{3} h$ \\
\hline
\end{tabular}

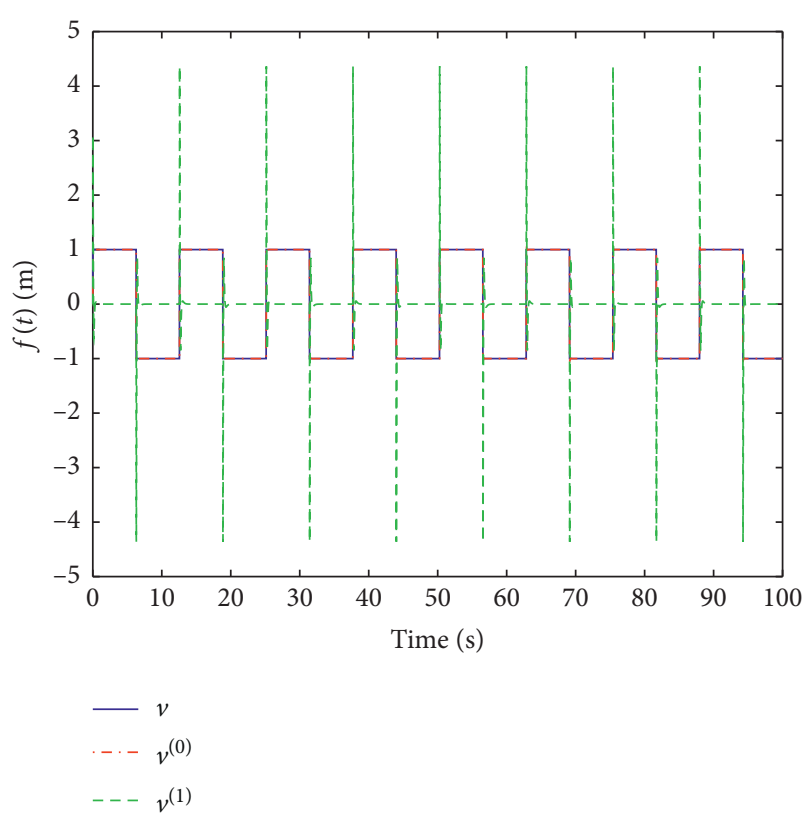

Figure 4: TD trend chart of the square wave.

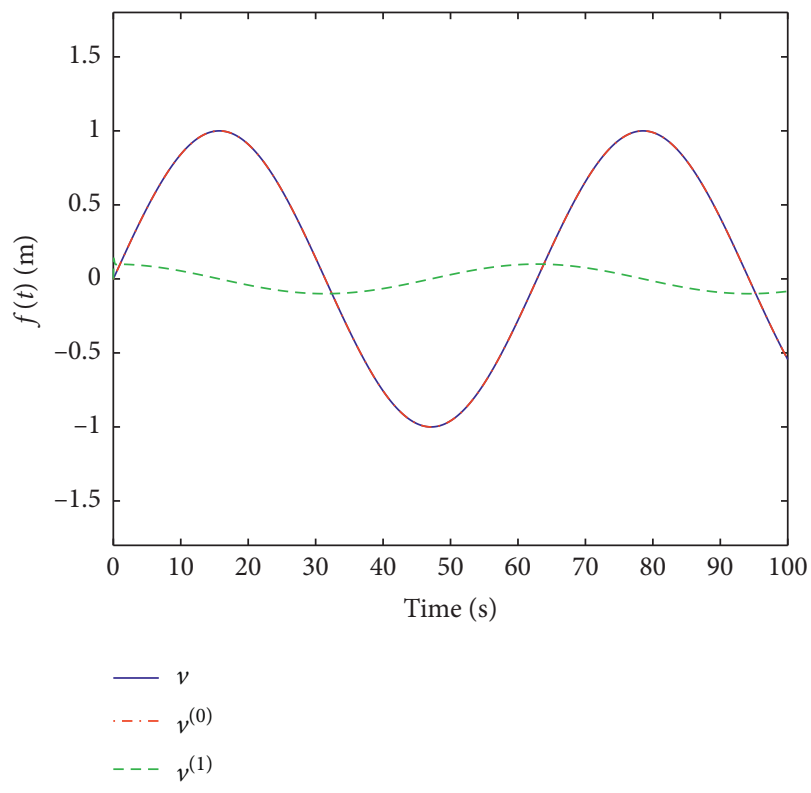

FIGURE 5: TD trend chart of $\sin (0.1 t)$. 


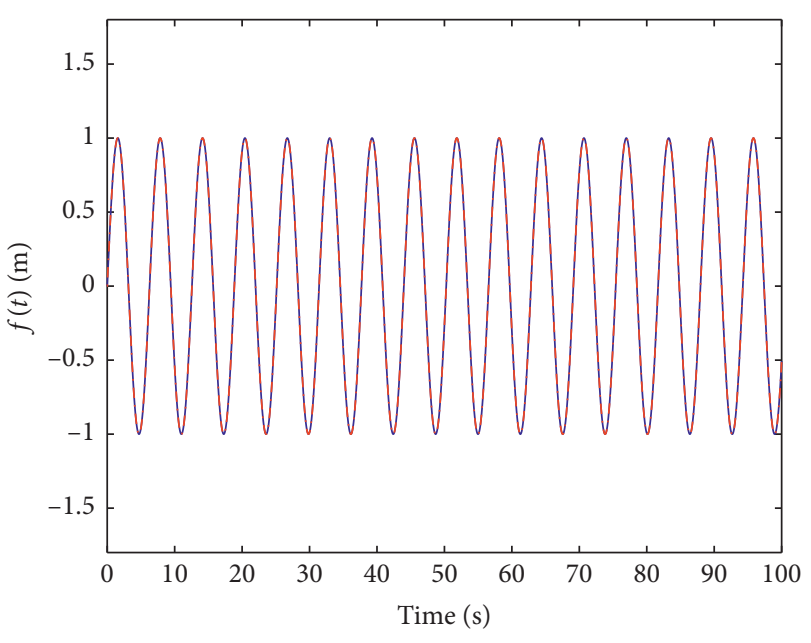

$-v$

Figure 6: TD trend chart of $\sin (t)$.

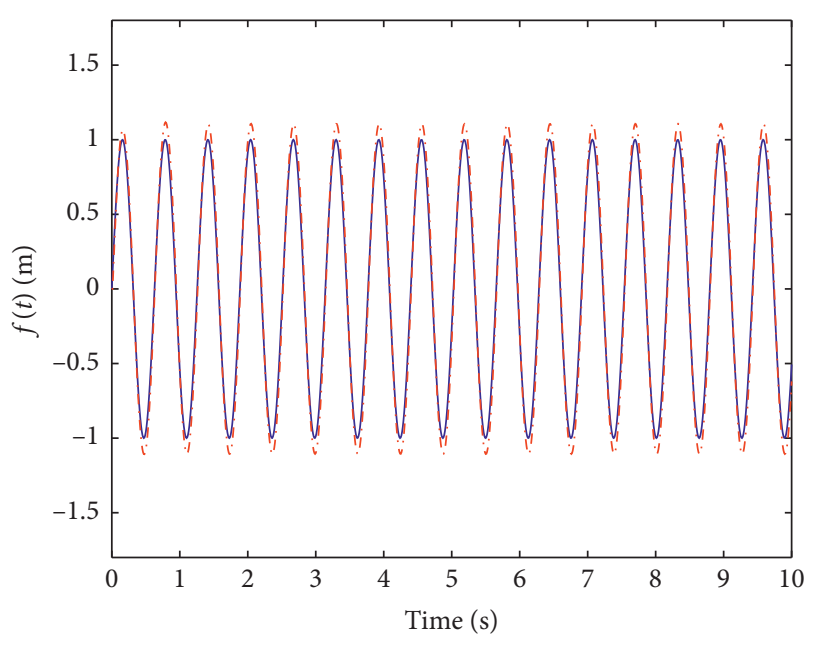

$-v$

FIgURE 7: TD trend chart of $\sin (10 t)$.

TABLe 3: ESO parameter table.

\begin{tabular}{lc}
\hline Item & \\
\hline$h$ & 0.01 \\
$\delta_{2}$ & $h$ \\
$\beta_{01}$ & $1 / 1^{0} h$ \\
$\beta_{02}$ & $1 / 3 h^{1} h$ \\
$\beta_{03}$ & $2 / 8 h^{2} h$ \\
$\beta_{04}$ & $5 / 13 h^{3} h$ \\
\hline
\end{tabular}

In Figure 9, the observer output state signal $z_{2}$ and the state signal to be observed $x_{4}$ are displayed.

In Figure 10, the observer output state signal $z_{3}$ and the total disturbance signal $z_{4}$ are displayed.

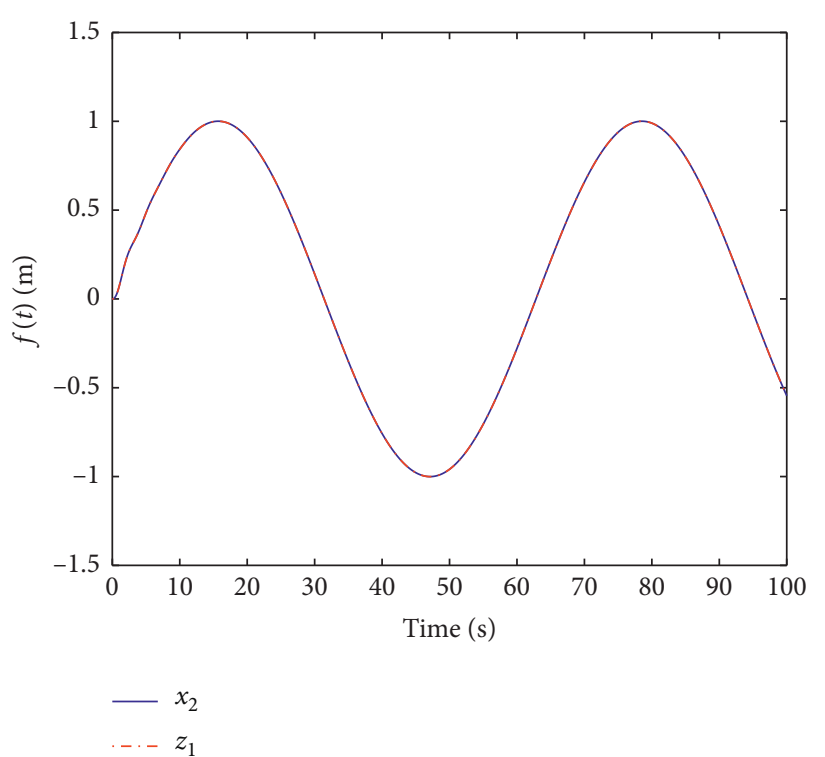

Figure 8: $z_{1}$ and $x_{2}$.

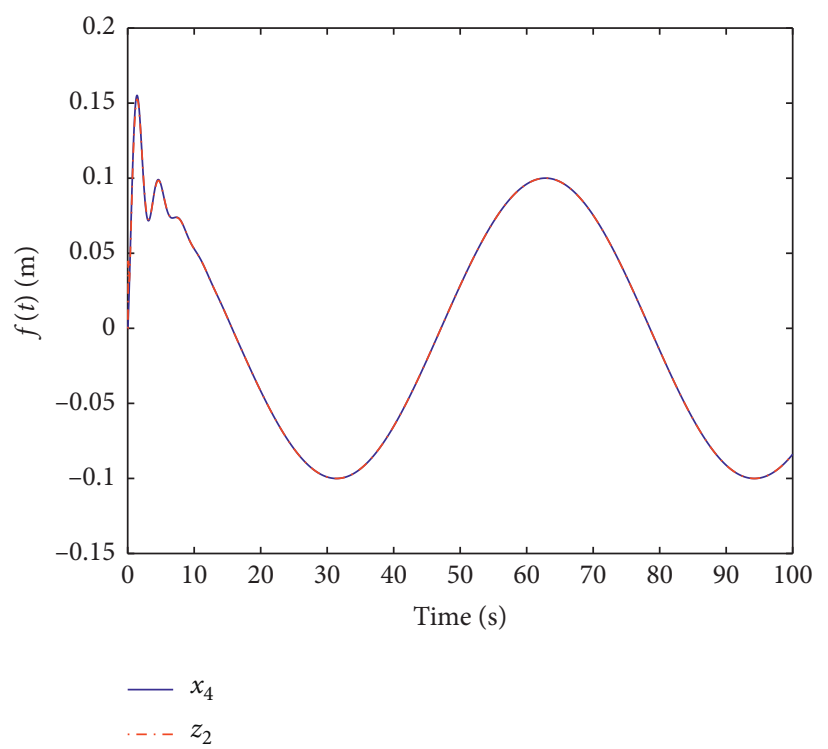

Figure 9: $z_{2}$ and $x_{4}$.

The simulation results show that the designed ESO estimation algorithm can estimate the state signal very accurately and the speed is also satisfactory.

4.3. Control Simulation. This paper uses the discretization form of complete control algorithm shown in equation (24). It not only analyzes the system response curve when $v$ takes the step signal, sine signal, and square wave signal, respectively, but also analyzes the system response curve when the disturbance signal $d_{1}$ and $d_{2}$ are added, respectively, and verifies the correctness and accuracy of the algorithm. The values of the LSEF parameters in the algorithm are shown in Table 4. 


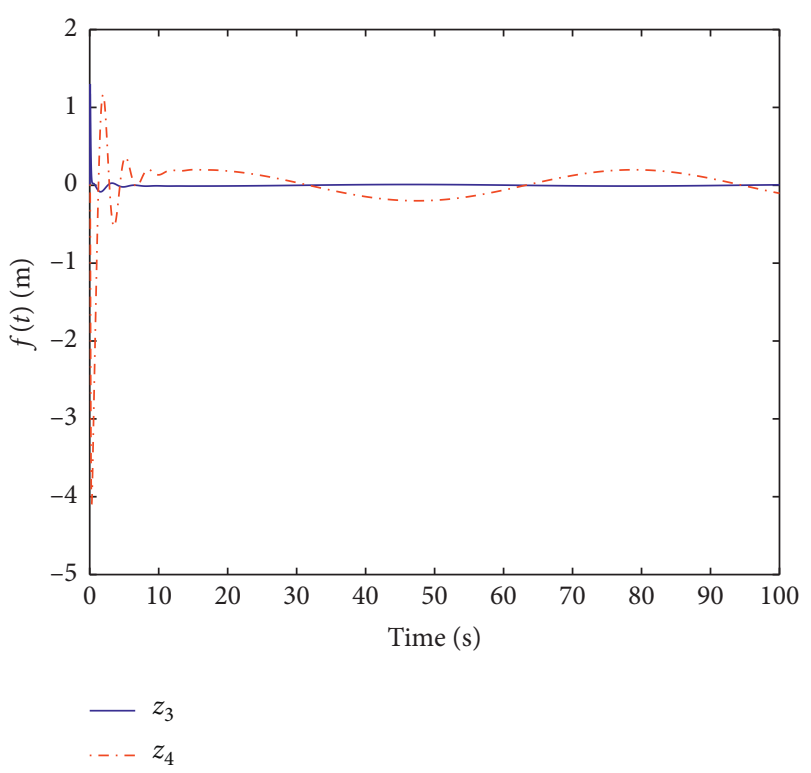

FIgURE 10: $z_{3}$ and $z_{4}$.

TABLE 4: LSEF parameter table.

\begin{tabular}{lc}
\hline Item & \\
\hline$h$ & 0.01 \\
$b_{0}$ & 10 \\
$\beta_{1}$ & 100 \\
$\beta_{2}$ & 300 \\
$\beta_{3}$ & 180 \\
\hline
\end{tabular}

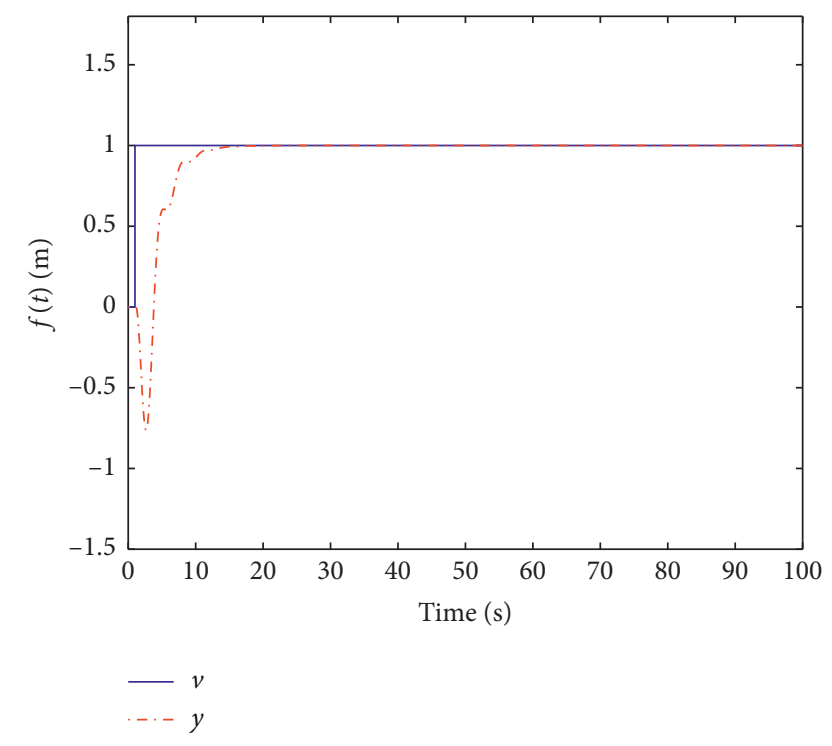

FIGURE 11: Control system response of $1(t)$.

When the input signal $v$ is the unit step signal $1(t)$, the output response of the system is shown in Figure 11.

When the input signal $v$ is a step signal $3(t)$, the output response of the system is shown in Figure 12.

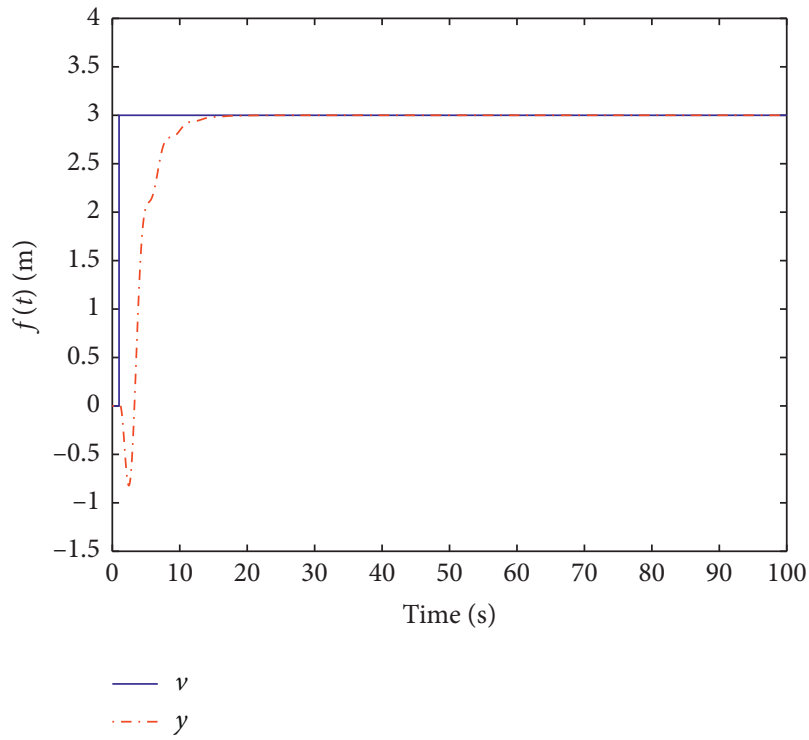

Figure 12: Control system response of 3(t).

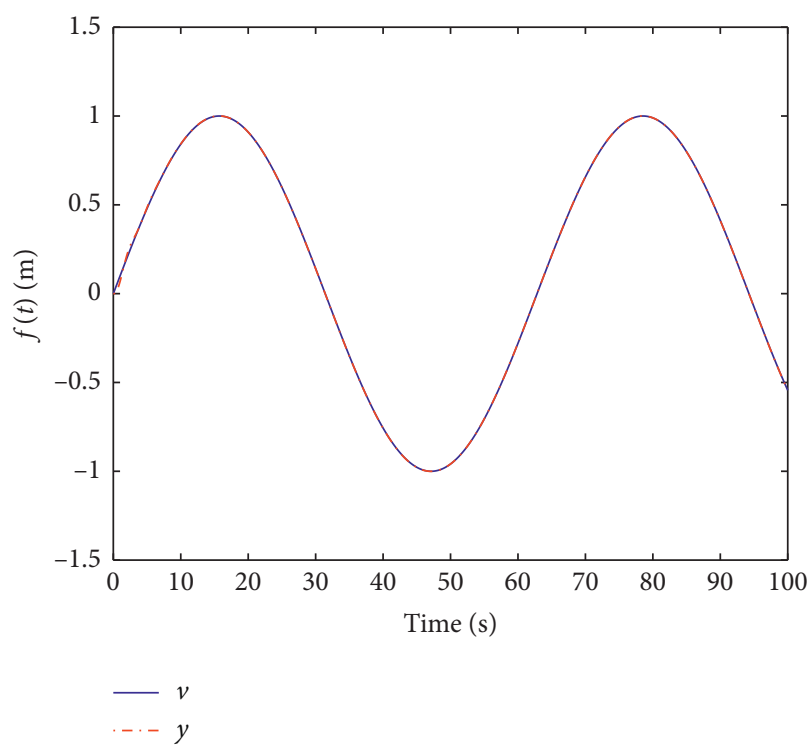

Figure 13: Control system response of $\sin (0.1 t)$.

When the input signal $v$ is a sinusoidal signal $\sin (0.1 t)$, the output response of the system is shown in Figure 13.

When the input signal $v$ is a periodic square wave signal $\operatorname{sign}(\sin (0.1 t))$, the system output response is as shown in Figure 14.

The periodic square wave signal has a severe impulse in the initial stage of the signal. The error signal $e_{i}$ between the differential signal $v_{i}$ of each order calculated by TD and the state signal $z_{i}$ observed by ESO deviates greatly, and the control force is strong, resulting in a larger spike. In a relatively short period of time, the system tracks and reaches the input signal value under the action of the control force, and there is basically no oscillation during the whole process, thereby suppressing the vibration. 


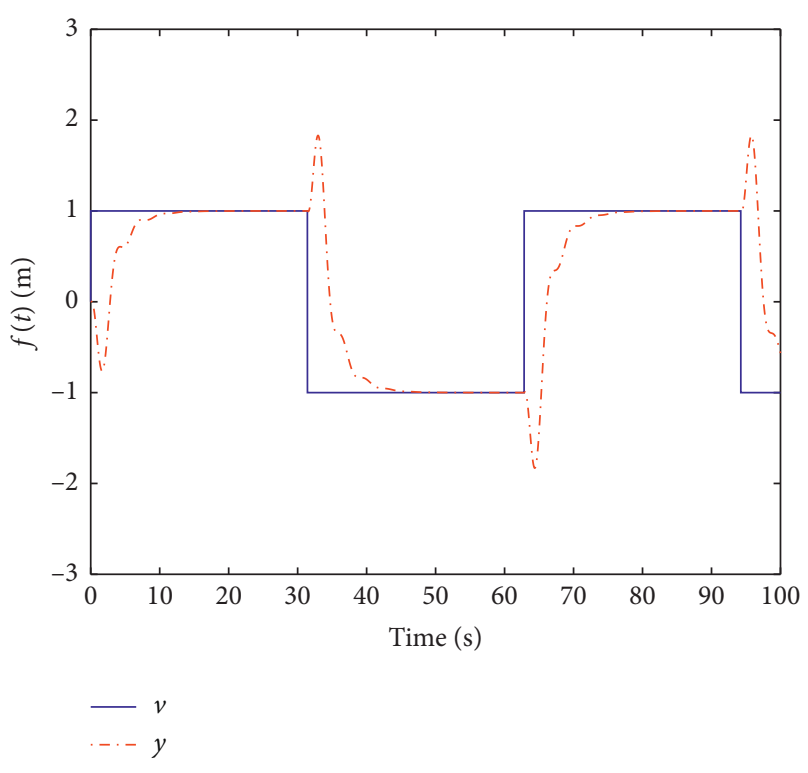

Figure 14: Control system response of $\operatorname{sign}(\sin (0.1 t))$.

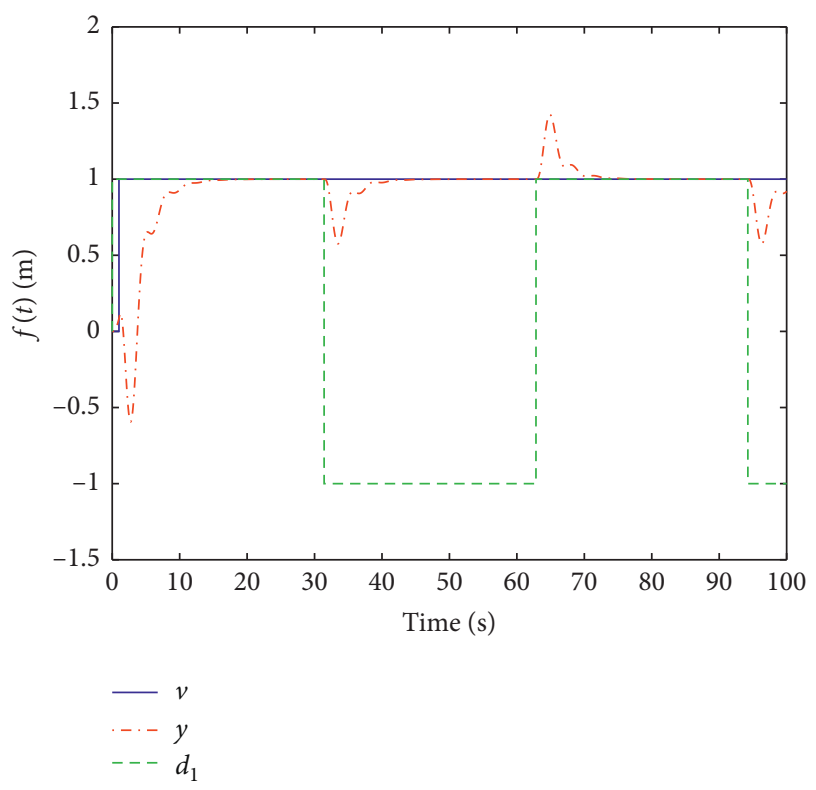

Figure 15: Control system response with disturbance signal $d_{1}$.

The input signal $v$ is the unit step signal $1(t), d_{2}=0$. The output response of the system is shown in Figure 15 when the square wave disturbance signal $d_{1}$ is added.

The input signal $v$ is the unit step signal $1(t), d_{1}=0$. The system output response is shown in Figure 16 when the square wave disturbance signal $d_{2}$ is added.

The simulation results of the complete control algorithm show that after the control system has set the control parameters, the controlled output $y$ can quickly track the trajectory of the system setting value $v$ without oscillation, thereby suppressing jitter, and its accuracy and robustness are also satisfactory.

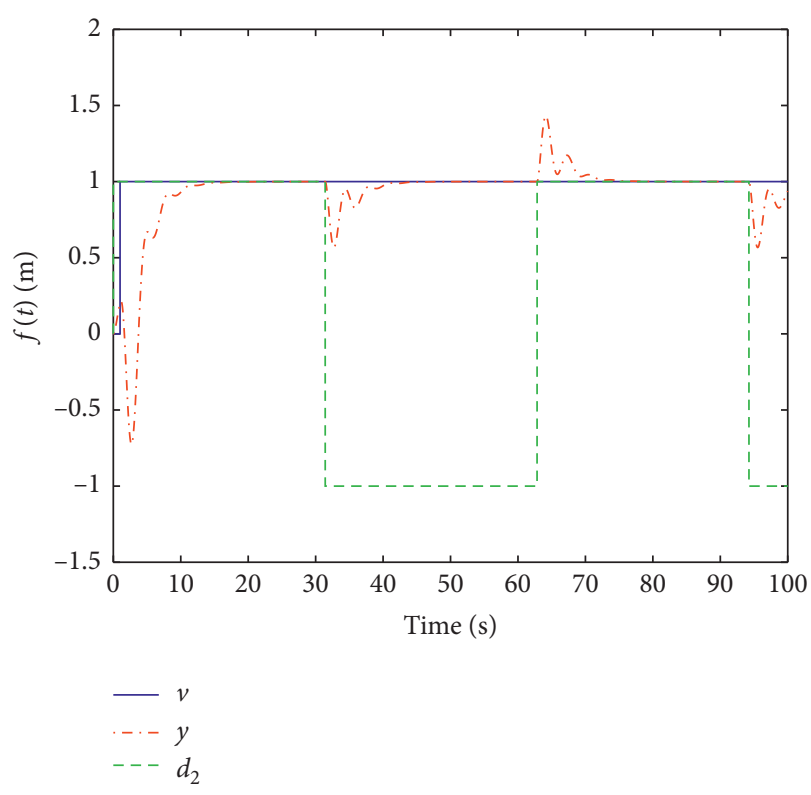

Figure 16: Control system response with disturbance signal $d_{2}$.

\section{Conclusion}

Aiming at the ropeway cable car vibration problem, this paper derives the nonlinear state space representation form in the general sense based on the two-mass-spring system model of translational vibration. Combined with the established representation model, this paper analyzes the relative order of the system, which provides a basis for the order of the NLADRC algorithm. On this basis, this paper establishes the NLADRC structure and demonstrates the corresponding algorithm from the three aspects of TD, ESO, and LSEF in combination with theoretical analysis. The complete algorithm of the NLADRC controller for suppressing the vibration of the cable car is obtained. Finally, this paper verifies the feasibility and accuracy of the algorithm through simulation. The follow-up work will further analyze the movement characteristics of the cable car on the basis of suppressing the vibration of the cable car and is committed to applying the research results of this paper to the actual system.

\section{Data Availability}

No data were used to support this study.

\section{Conflicts of Interest}

The authors declare that there are no conflicts of interest.

\section{Acknowledgments}

This work was supported by the Shandong Provincial Key Research and Development Project (2019GGX101005), Shandong Provincial Natural Science Foundation (ZR2017MF048), and National Natural Science Foundation of China (61803216). 


\section{References}

[1] B. Wie and D. S. Bernstein, "A benchmark problem for robust control design," Journal of Guidance, Control, and Dynamics, vol. 15, no. 5, pp. 1057-1059, 1992.

[2] B. Wie and D. S. Bernstein, "Benchmark problems for robust control design (1992 ACC version)," in Proceedings of the IEEE 1992 American Control Conference, Chicago, IL, USA, June 1992.

[3] S. Wang, J. Na, and Y. Xing, "Adaptive optimal parameter estimation and control of servo mechanisms: theory and experiments," IEEE Transactions on Industrial Electronics, p. $1,2020$.

[4] S. Wang and J. Na, "Parameter estimation and adaptive control for servo mechanisms with friction compensation," IEEE Transactions on Industrial Informatics, vol. 16, no. 11, pp. 6816-6825, 2020.

[5] S. Wang, L. Tao, Q. Chen, J. Na, and X. Ren, "USDE-based sliding mode control for servo mechanisms with unknown system dynamics," IEEE/ASME Transactions on Mechatronics, vol. 25, no. 2, pp. 1056-1066, 2020.

[6] Q. Chen, H. Shi, and M. Sun, "Echo state network-based backstepping adaptive iterative learning control for strictfeedback systems: an error-tracking approach," IEEE Transactions on Cybernetics, vol. 50, no. 7, pp. 3009-3022, 2020.

[7] Q. Chen, S. Xie, M. Sun, and X. He, "Adaptive nonsingular fixed-time attitude stabilization of uncertain spacecraft," IEEE Transactions on Aerospace and Electronic Systems, vol. 54, no. 6, pp. 2937-2950, 2018.

[8] M. Tao, Q. Chen, X. He, and M. Sun, “Adaptive fixed-time fault-tolerant control for rigid spacecraft using a double power reaching law," International Journal of Robust and Nonlinear Control, vol. 29, no. 2, 2019.

[9] N. Jing, B. Wang, G. Li, S. Zhan, and W. He, "Nonlinear constrained optimal control of wave energy converters with adaptive dynamic programming," IEEE Transactions on Industrial Electronics, vol. 60, no. 10, pp. 7904-7915, 2019.

[10] N. Jing, Y. Huang, X. Wu, S.-F. Su, and G. Li, "Adaptive finitetime fuzzy control of nonlinear active suspension systems with input delay," IEEE Transact ions on Cybernetics, vol. 50, no. 6, pp. 2639-2650, 2019.

[11] N. Jing, A. S. Chen, Y. Huang et al., "Air-fuel ratio control of spark ignition engines with unknown system dynamics estimator: theory and experiments," IEEE Transactions on Control Systems Technology, no. 99, pp. 1-8, 2019.

[12] B. Sun, C. Yang, Y. Wang, W. Gui, I. Craig, and L. Olivier, "A comprehensive hybrid first principles/machine learning modeling framework for complex industrial processes," Journal of Process Control, vol. 86, pp. 30-43, 2020.

[13] X. Gao, C. Zhang, C. Zhu, and X. Ren, "Identification and control for hammerstein systems with hysteresis non-linearity," IET Control Theory \& Applications, vol. 9, no. 13, pp. 1935-1947, 2015.

[14] X. Gao, "Adaptive neural control for hysteresis motor driving servo system with bouc-wen model," Complexity, vol. 2018, Article ID 9765861, 9 pages, 2018.

[15] X. Gao and X. Hu, "A petri net neural network robust control for new paste backfill process model," IEEE Access, vol. 8, pp. 18420-18425, 2020.

[16] X. Gao, W. Zhao, S. Wang, M. Wang, and X. Ren, “A prescribed performance adaptive control for hysteresis hammerstein system," Journal of Systems Ence and Complexity, vol. 32, no. 4, pp. 1039-1052, 2019.
[17] T. Zeng, X. Ren, Y. Zhang, G. Li, and N. Jing, “An integrated optimal design for guaranteed cost control of motor driving system with uncertainty," IEEE/ASME Transactions on Mechatronics, vol. 24, no. 6, pp. 2606-2615, 2020.

[18] T. Zeng, X. Ren, and Y. Zhang, "Fixed-time sliding mode control and high-gain nonlinearity compensation for dualmotor driving system," IEEE Transactions on Industrial Informatics, vol. 16, no. 6, pp. 4090-4098, 2020.

[19] Y. Zhang, P. Stansby, and G. Li, "Non-causal linear optimal control with adaptive sliding mode observer for multi-body wave energy converters," IEEE Transactions on Sustainable Energy, p. 1, 2020.

[20] Y. Zhang, R. Vepa, G. Li, and T. Zeng, "Mars powered descent phase guidance design based on fixed-time stabilization technique," IEEE Transactions on Aerospace and Electronic Systems, vol. 55, no. 4, pp. 2001-2011, 2019.

[21] Y. Zhang, G.-F. Ma, Y.-N. Guo, and T.-Y. Zeng, "A multi power reaching law of sliding mode control design and analysis," Acta Automatica Sinica, vol. 42, no. 3, pp. 466-472, 2016.

[22] Q. Chen, X. Yu, M. Sun, C. Wu, and Z. Fu, "Adaptive repetitive learning control of PMSM servo systems with bounded nonparametric uncertainties: theory and experiments," IEEE Transactions on Industrial Electronics, p. 1, 2020.

[23] J. Han, "From PID to active disturbance rejection control," IEEE Transactions on Industrial Electronics, vol. 56, no. 3, pp. 900-906, 2009.

[24] H. Zhang, S. Zhao, and Z. Gao, "An active disturbance rejection control solution for the two-mass-spring benchmark problem," in Proceedings of the 2016 American Control Conference (ACC), Boston, MA, USA, July 2016.

[25] S. Li, J. Li, and Y. Mo, "Piezoelectric multimode vibration control for stiffened plate using ADRC-based acceleration compensation," IEEE Transactions on Industrial Electronics, vol. 61, no. 12, pp. 6892-6902, 2014.

[26] Z. Gao, "Scaling and bandwidth-parameterization based controller tuning," in Proceedings of the 2003 American Control Conference, 2003, pp. 4989-4996, Denver, CO, USA, June 2003.

[27] R. Miklosovic, A. Radke, and Z. Gao, "Discrete implementation and generalization of the extended state observer," in Proceedings of the 2006 American Control Conference, Minneapolis, MN, USA, June 2006.

[28] S. Zhao and Z. Gao, "An active disturbance rejection based approach to vibration suppression in two-inertia systems," in Proceedings of the 2010 American Control Conference, pp. 1520-1525, Baltimore, MD, USA, June 2010.

[29] R. Madonski, S. Shao, H. Zhang, Z. Gao, J. Yang, and S. Li, "General error-based active disturbance rejection control for swift industrial implementations," Control Engineering Practice, vol. 84, pp. 218-229, 2019.

[30] Z.-L. Zhao and B.-Z. Guo, "A nonlinear extended state observer based on fractional power functions," Automatica, vol. 81, pp. 286-296, 2017.

[31] B. Wang, J. Yang, H. Jiao, K. Zhu, and Y. Chen, "Design of auto disturbance rejection controller for train traction control system based on artificial bee colony algorithm," Measurement, vol. 160, Article ID 107812, 2020.

[32] J. Han, "Nonlinear PID controller," ACTA Automation Sinica, vol. 20, no. 4, pp. 487-490, 1994.

[33] Y.-Q. Wang, G. Ren, and J.-D. Zhang, "Anti-windup schemes for nonlinear active disturbance rejection control in marine steam turbine," Journal of Marine Science and Technology, vol. 24, no. 1, pp. 47-53, 2016. 
[34] G. Sun, Y. Li, W. Jin, and L. Bu, "A nonlinear three-phase phase-locked loop based on linear active disturbance rejection controller,” IEEE Access, vol. 5, pp. 21548-21556, 2017.

[35] J. Li, Y. Xia, X. Qi, and Z. Gao, "On the necessity, scheme, and basis of the linear-nonlinear switching in active disturbance rejection control," IEEE Transactions on Industrial Electronics, vol. 64, no. 2, pp. 1425-1435, 2017.

[36] R. Wang, X. Li, Y. Liu, W. Fu, S. Liu, and X. Ma, "Multiple model predictive functional control for marine diesel engine," Mathematical Problems in Engineering, vol. 2018, no. 1, 18 pages, Article ID 7641862, 2018. 